\title{
Application of weight function method in the assessment of crack propagation through stiffened panel
}

\author{
I. Gledić \& J. Parunov \\ Faculty of Mechanical Engineering and Naval Architecture, Zagreb, Croatia
}

\begin{abstract}
The purpose of this paper is to examine the weight function method as rapid stress intensity calculation method for damaged stiffened panel. Typical application is the crack propagation assessment when the ship is damaged due to collision or grounding. If the stress distribution is known, as well as the weight function for specific crack geometry it is possible to calculate the stress intensity factor for any non-linear stress distribution. The method is firstly verified on the examples when analytical solutions are available. A new method to account for the effect of stiffener along the crack path using the weight function method is then proposed. The applicability of the weight function method for crack propagation through the damaged stiffened panel is also investigated.
\end{abstract}

\section{INTRODUCTION}

Structural elements contain flaws and discontinuities due to welding. Under loading those flaws can become cracks that can propagate to fracture. Most of the cracks are detected during a survey and inspection. Detected cracks are often too small and can be ignored, but larger cracks can propagate faster and cause serious damage. Classification societies have developed procedures for fatigue design by SN approach (IACS 2012). However, prediction of crack growth rate is very difficult.

Few experiments were conducted to develop models that can predict crack growth rate of long cracks (Dexter et al. 2005). Experiment with a single non-redundant stiffened panel showed that long cracks propagate in a stable manner (Dexter \& Mahmoud 2004). The experiment also showed a decrease of crack growth rate between stiffeners. Even though long cracks propagated in the stable manner, it was not clear how to predict crack propagation rate for welded structures like stiffened panels.

Ship structure can be damaged by collision or grounding. During the towing period of the ship from the place of accident to repair facility damage can progress very fast as fatigue crack and cause hazard. For calculation of ship structural strength is very important to make a rapid assessment of Stress Intensity Factor (SIF) (Bardetsky 2013).

The assessment of damage growth is very important during towing period. It can be assumed that during this period crack propagates in a similar way as would ordinary fatigue crack. Damage growth assessment can be performed by the fracture mechanics approach. The crack growth rate is defined as a function of the stress intensity factor at the crack tip. Fluctuating stresses enforcing crack to propagate are caused by the wave bending moments. With defined stress range and geometry function, crack propagation curve can be calculated by numerical integration of Paris-Erdogan law (Bužančić Primorac \& Parunov 2013).

There are many methods for calculating stress intensity factor. Serror \& Marchal (2009) proposed method that takes into the account the analysis of simultaneous crack propagation. This technique avoids successive re-meshing along the crack path. A line spring method coupled with a fitted structural stiffness condensation method is used to calculate the stress intensity factors. The idea of the line spring method was to analyze the part-through crack as several single edged specimens, the cracked structure part. The crack is defined as a line of couple of nodes, with the same coordinates, and separated. The fundamental concept is that the perfect knowledge of the crack lip displacement makes possible to determine the stress intensity factor. For every crack depth of each node along the crack line, stress intensity factors are calculated according to the loads acting on the structure. With this methodology, the propagation of a crack is not idealized as it is when analytical expressions are used. The propagation line follows its own path. This approach was used to asses fatigue crack propagation on a welded connection between the bracket and longitudinal stiffener.

Marchal et al. (2009) implemented same methodology for assessment of fatigue crack propagation 
on a welded connection between the side shell and longitudinal stiffener.

The SIF can be obtained by implementing the contour integral method available in some FE software (Dexter \& Pilarski 2000). The contour integral in linear elastic fracture mechanics characterizes the energy release associated with crack propagation, and is calculated using any closed ring of the elements encircling the crack tip. The problem with the contour integral method is that the mesh size in the vicinity of the crack tip is required to be as fine as possible and fine mesh takes time to be modeled.

The fracture behavior can be also modeled by FE with the VCCT (Virtual Crack Closure Technique) for the calculation of the strain energy release rate $(G)$, and, in turn, the calculation of the SIF (Kwon et al. 2010). This technique captures the effect of stiffener. All methods listed are time consuming and impractical for rapid crack propagation assessment.

A more simplified method for SIF calculation is a weight function method (Carroll et al. 2003). The biggest advantage of this method is that it depends only upon loading and geometry. The unique feature of the weight function method is that once the weight function for a particular cracked body is determined, the stress intensity factor for any loading system applied to the body can be calculated. The weight function method has potential benefits for allowing rapid assessment of damaged steelplated structure that would otherwise require the use of high fidelity modeling of the entire structures, such as through the use of FE analysis.

The aim of this work is to examine whether the weight function method can be used as a rapid SIF calculation method in case when the ship is damaged in collision or grounding. In order to verify method, the method is firstly compared with analytical solution for crack on one side and crack in the center of a finite width unstiffened plate. Secondly, crack propagation through stiffened panel is analyzed for the case when analytical solution is available. In the final step of research validation of results is made in two FE models of damaged panels.

\section{WEIGHT FUNCTION METHOD}

This method enables SIF calculations for a variety of loading conditions and the crack length $a$ by simple integration of the weight function $m(x, a)$ and the distribution of stress $\sigma(x)$, normal to the plane $x$ of the prospective crack plane:

$K=\int_{0}^{a} \sigma(x) m(x, a) d x$
General weight function expression (Shen \& Glinka 1991), which can be used for a wide variety of one-dimensional mode I cracks, is given as follows:

$$
=\frac{2}{\sqrt{2 \pi(a-x)}}\left[\begin{array}{c}
1+M_{1}\left(1-\frac{x}{a}\right)^{\frac{1}{2}}+M_{2}\left(1-\frac{x}{a}\right) \\
+M_{3}\left(1-\frac{x}{a}\right)^{\frac{3}{2}}
\end{array}\right]
$$

where: $a$ is a crack size; $x$ is a crack coordinate; $M_{1}, M_{2}, M_{3}$ are parameters appropriate for a crack geometry.

Before calculating $\operatorname{SIF}(K)$, few steps must be followed:

a. Definition of crack propagation geometry in finite width plate as edge crack, central through thickness crack, double-edge cracks, semielliptical surface cracks;

b. Calculation of the $M_{1}, M_{2}$ and $M_{3}$ coefficients (Carroll et al. 2003) applicable for that particular crack geometry;

c. Determination of stress distribution $\sigma(x)$ in the prospective crack plane through structural analysis techniques (FE analysis);

d. Integration of the product of weight function $m(x, a)$ and stress distribution $\sigma(x)$ over the entire crack length $a$, using equation (1).

This approach can be used for calculating stress intensity factors for any non-linear stress distribution providing that the stress function, $\sigma(x)$, is known. Very often the stress distribution is obtained numerically by using the finite element method. It should be mentioned that the weight function presented in the report (Carroll et al. 2003) does not account for out of plane bending.

\section{EXAMPLES OF CRACK PROPAGATION ANALYSIS}

This work is limited only on two cases: edge crack propagation and central through thickness propagation. For the validation of results two assumptions were made. First, the plate is uncracked and second, the stress distribution is uniform along the plate.

\subsection{Analytical solution vs. weight function}

The stress intensity factor $K$ is the principal governing parameter for a crack growth in the linear fracture mechanics approach. It incorporates the 
effects of the stresses and the crack size within the crack tip zone. A general expression of stress intensity factor $K$ is given as follows:

$$
K=F \sigma \sqrt{\pi u}
$$

where: $a$ is a crack size; $\sigma$ stress away from the crack; $F$ is a joint geometry function depending on the loading and configuration accounting for cracked body size and shape (Koiter 1965).

Results of $K$ obtained from the analytical solution for unstiffened plate with crack on one side and central through thickness crack were compared with weight function results of $K$. Analysis showed (Fig. 1) that weight function gives slightly overestimated results for $K$. The difference between results for edge crack is less than $2 \%$. In case of central through thickness crack difference between the results is around $6 \%$.

This comparison confirmed a good agreement between results and proved that weight function is appropriate for further analysis.

\subsection{Linear stress distribution}

The purpose of this section is to show the application of the weight function for the case when the analytical solution for SIF is not available. Such case is the linear stress distribution along the edge of unstiffened plate. The stress varies along the plate edge from value $\sigma_{A}$ on one corner to the value $\sigma_{B}$ on the other corner (Fig. 2).

Analytical solution for $K$ is available only when loading is uniform. For the comparison of these two methods in case of linear stress distribution the analytical solution of $K$ is calculated for mean stress linear distribution. It is assumed that plate is unstiffened and uncracked.

The linear stress distribution is applied along the assumed crack propagation line. It is shown in Figure 3 that the weight function method and

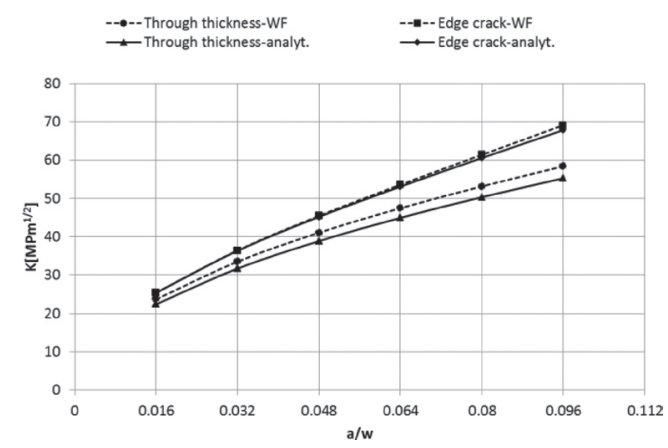

Figure 1. Comparison of results of SIF (analytical vs. weight function solution).

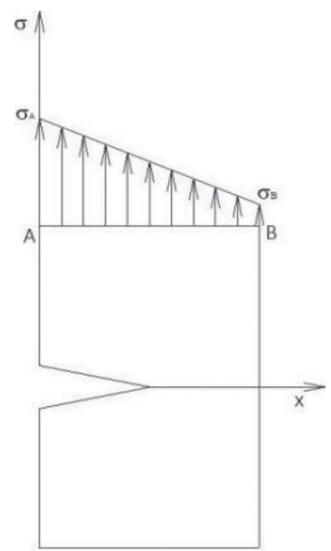

Figure 2. Linear stress distribution in case of one edge crack in unstiffened panel.

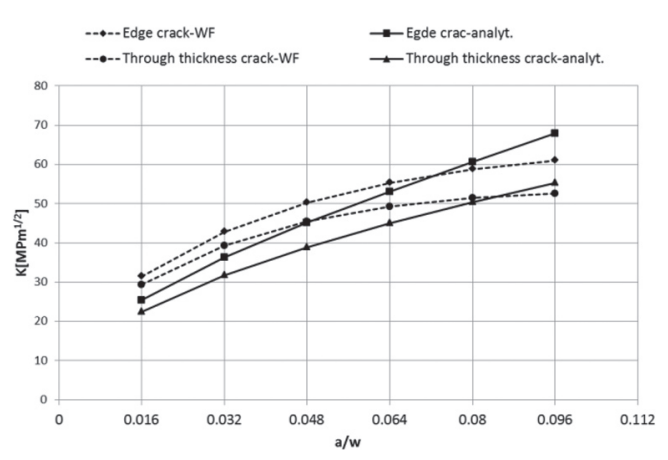

Figure 3. Comparison of results of SIF for linear stress distribution (weight function solution) and SIF for mean stress distribution (analytical solution).

analytical solution provide good agreement, but the weight function method gives a better estimation of $K$ because it accounts for linear stress distribution.

\subsection{Stiffened panel}

Large scale experiments on stiffened panels are rarely conducted because they present physical and economic issues that often limit the scope that testing may encompass. Many experiments verified the stability of long crack propagation in redundant structures (Dexter \& Pilarski 2002). The experiment showed that welded stiffeners will slightly slow down an approaching crack. When the cracks encountered the stiffeners, the rate of propagation through the web of the stiffener was similar to the rate of propagation in the plate beyond the stiffener.

The weight function method does not account for crack propagation through the web of stiffener. 
For application of the weight function method on stiffened panels, calculation of correction factors was proposed to account stiffener effect (Carroll et al. 2003). Even with the correction factors, SIF was still much higher than the results obtained through finite element method. The approach seemed to promise, but further research is required.

To account stiffener effect in weight function method for calculation of SIF a new approach is proposed. It is assumed that stiffener has a web height $h$. The web height $h$ equals the range of the linear increase of the plate thickness. The range represents the length between point $\mathrm{A}$ and point $\mathrm{B}$. From point $\mathrm{B}$ to point $\mathrm{C}$ plate thickness linearly decreases.

The relation between the plate thickness in point $\mathrm{B}$ and stiffener height is given as follows:

$t^{\prime}=\frac{A}{h}=\frac{A_{f}+A_{w}}{h}$

where: $h$ is the height of stiffener; $A$ is the total area of stiffener (flange and web). See Figure 4.

The linear increase of the plate thickness from point A to point B results in the linear decrease of the stress distribution as it is shown in Figure 5. The effect of welding is disregarded in this work.

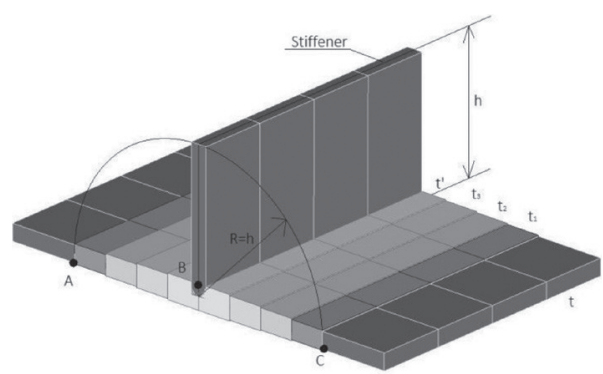

Figure 4. Proposed method for linear plate thickness distribution.

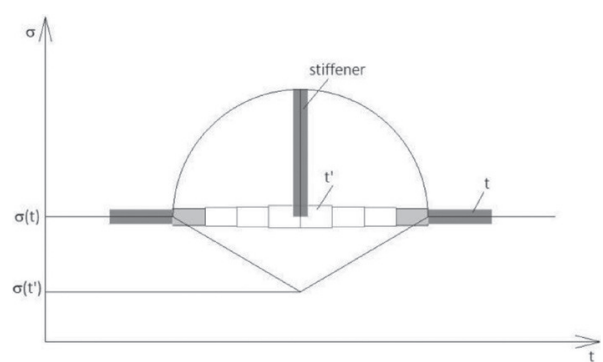

Figure 5. Linear stress distribution in case of linear plate thickness distribution.
Figure 6 shows the comparison of the normalized $K$ values obtained analytical solution for a panel with integral stiffeners (Dexter et al. 2005) and normalized $K$ values for stiffened panel calculated by the weight function method. The normalized $K$ value equals:

$\frac{K}{\sigma \sqrt{\pi a}}$

where: $K$ is the stress intensity factor for crack length $a ; \sigma$ is mean stress value of linear stress distribution.

Analytical solution of normalized $K$, proposed by Dexter et al. (2005) shows that the normalized $K$ increases linearly between the solution for intact stiffener and a completely severed stiffener. Between point on the panel where stiffener is located and the point equal to the height of the stiffener web, linear interpolation is made.

Using the proposed new method of linear plate thickness distribution, normalized $K$ is calculated by simple integration of weight function method and linear stress distribution due to linear plate thickness increase. As the crack propagates through the plate and approaches to a point of linear increase of the plate thickness, normalized SIF curve decreases. It continues to decrease rapidly until it reaches the final plate thickness that is stiffener web height. When the stiffener is completely severed, crack continues to propagate rapidly until it reaches second stiffener. After the crack reaches second stiffener the scenario repeats until crack propagates through the whole plate width.

The comparison of the results showed a good agreement (Fig. 6). However, it is important to emphasize that comparison of the results is only qualitative. Plate width has a great influence on the value of normalized $K$. As mentioned before, the

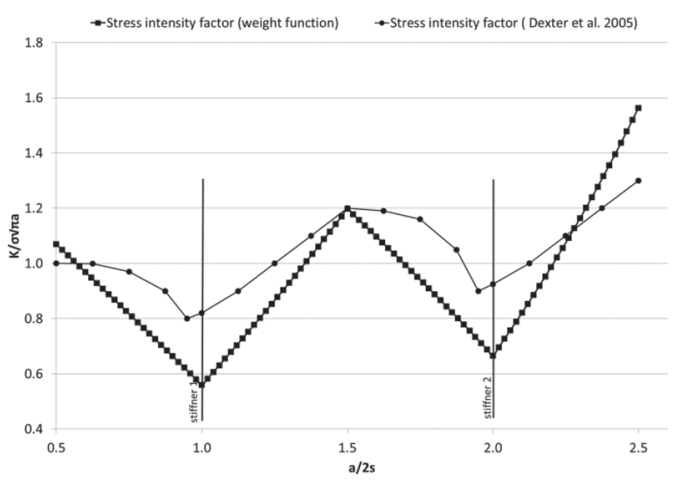

Figure 6. SIF results for stiffened panel compared to results for a panel with integral stiffeners (Dexter et al. 2005). 
weight function expression depends upon crack length and crack coordinate, but also it depends upon geometry parameters $M_{1}, M_{2}$ and $M_{3}$. The three parameters are functions of ratio of crack length and plate width. As the crack propagates, it can be seen in Figure 6 that, near the end of the plate, normalized $K$ value significantly increases over analytical results. The ratio of crack length and plate width near the end of plate is approximately 1 . At this point three parameters reach their maximum value, and thus the value of the weight function significantly increases.

\section{APPLICATION TO DAMAGED STIFFENED PANEL}

Longitudinal stiffened panels on ship decks and offshore structures are subjected to axial compression and tension due to sagging and hogging moments.

In this work damage is simplistically modeled as a circular opening, according to Underwood et al. 2012. The finite element model was modeled using FEMAP software in order to calculate the stress distribution along assumed crack path. A unit panel consisting of a portion of the plate of width $b$ with a stiffener centered on the plate strip provided with circular opening $d$ in the center of the panel. As it is symmetrical with respect to the unit panel, only a half portion of the circular opening is considered in the analysis. The yield strength of plate $\left(\sigma_{y}\right)$ is $250 \mathrm{~N} / \mathrm{mm}^{2}$ with Young's modulus of elasticity $(E)$ as $210 \mathrm{~N} / \mathrm{mm}^{2}$ and Poisson's ratio $(v)$ of 0.3 . The length of the panel and the thickness of the plate is taken as $1500 \mathrm{~mm}$ and $6 \mathrm{~mm}$, respectively. For the present study the chosen width of plate is $510 \mathrm{~mm}$. Mesh size of finite element model is $25 \times 25 \mathrm{~mm}$. The size of the circular opening equals half the size of plate width, $255 \mathrm{~mm}$.

Along the crack propagation line more refined mesh $12,75 \times 12,75 \mathrm{~mm}$ was used in both examples (Fig. 7 \& Fig. 8).

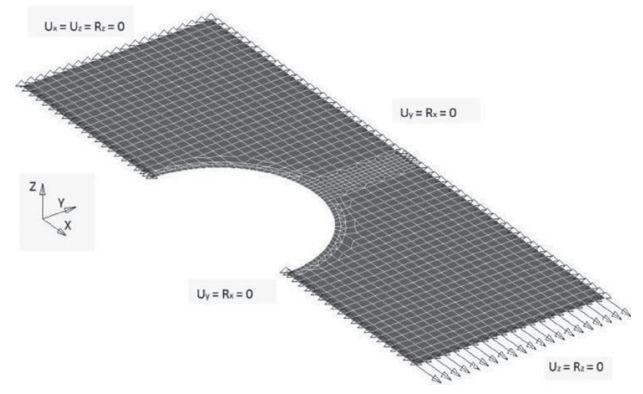

Figure 7. Un-stiffened finite element model.
The stress range distribution obtained from the first finite element model (unstiffened panel) was applied as a loading upon second finite element model (Fig. 7). For second finite element model (Fig. 8) selected stiffener, according to ISA (Unequal Indian Standard Angles), is 50306 which denotes an unequal Indian standard angle of flange width $30 \mathrm{~mm}$, with overall web depth of $50 \mathrm{~mm}$ and uniform thickness of section $6 \mathrm{~mm}$. The stiffener was modeled as proposed in chapter 3.3. According to equation (4) final thickness of plate is $8,88 \mathrm{~mm}$.

In the area of modified plate thickness in stiffened model and along the assumed crack path, more refined mesh was applied. Each model is subjected to axial load $\left(P_{x}\right)$ on one end and are simply supported on the opposite end. Rotation about the longitudinal direction is prevented at all nodes along the longitudinal edges with the displacement along the same edges allowed to freely wave in, allowing for continuity between adjacent stiffened plates. Rotation about axis $z$ as well as the displacement along axis $z$ is prevented.

After structural analysis in FEMAP, stress distribution along the crack propagation line is obtained. Stress intensity factor was calculated by

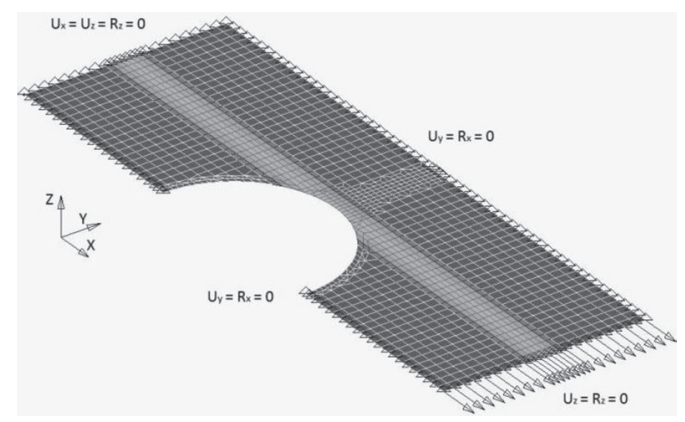

Figure 8. Stiffened finite element model.

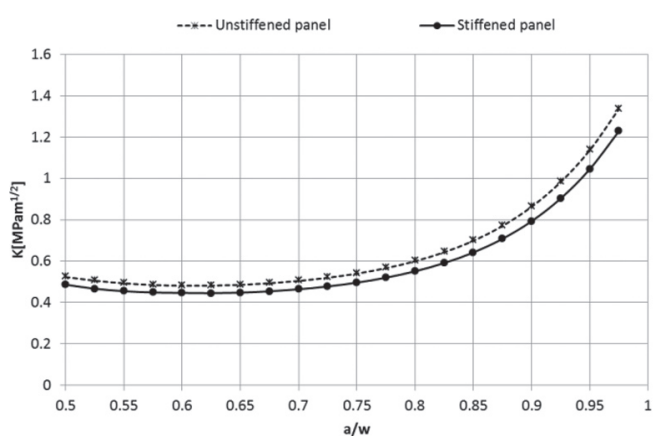

Figure 9. Comparison of SIF results for stiffened and unstiffened panel. 
integrating linear stress distribution and weight function along the crack propagation line. Results show (Fig. 9) that stress intensity factor increase in both cases at the same rate, but the in the case of stiffened panel stiffener reduces the value stress intensity factor between $7,5 \%$ and $9 \%$.

\section{CONCLUSION}

The aim of this paper is an initial verification of the weight function method for rapid assessment of the SIF for damaged ship.

Two cases are studied for the intact unstiffened plate, crack on one side and through thickness central crack. When uniform stress distribution is applied, comparative analysis indicates that both methods, the weight function method and the analytical approach, provide fairly close results. When linear stress distribution is applied, analytical results are not available. In that case, a solution for SIF can be easily obtained by the weight function method. The results look promising, but further verification is necessary.

The weight function method does not account for the stiffener height in the case of the intact stiffened panel. In this work the range of the linear increase of the plate thickness equals to the web height of the stiffener is proposed, and same for a decrease of the plate thickness. The proposed method of linear change of the plate thickness provided quantitatively good results when compared to the results of SIF from Dexter et al. (2005).

The method is then applied to damaged unstiffened and stiffened panels. It is shown by the weight function method that stiffener causes only small reduction of the SIF.

The weight function method may be considered as rapid SIF assessment for damaged ship, but it is necessary to perform further verification, preferably using FEM.

\section{ACKNOWLEDGMENT}

This work has been fully supported by Croatian Science Foundation under the project 8658.

\section{REFERENCES}

Bardetsky, A. 2013. Fracture mechanics approach to assess the progressive structural failure of a damaged ship, Collision and Grounding of Ships and Offshore Structures, Edts. Amdhal, J., Ehlers, S., J. Leira, B., Taylor \& Francis Group, pages 77-84. London.
Bužančić Pimorac, B. \& Parunov, J. 2013. Reduction of the Ultimate Strength due to Crack Propagation in Damaged Ship Structure, 15th International Congress of the International Maritime Association of the Mediterranean, Spain.

Carroll, L.B., Tiku, S. \& Dinovitzer, A.S. 2003. Rapid Stress Intensity Factor Solution Estimation for Ship Structure Applications, Ship Structure Committee SSC-429, Washington.

Dexter, R.J. \& Pilarski, P.J. 2000. Effect of Welded Stiffeners on Fatigue Crack Growth Rate, Ship Structure Committee SSC-413, Washington.

Dexter, R.J. \& Pilarski, P.J. 2002. Crack Propagation in Welded Stiffened Panels, Journal of Constructional Steel Research, Volume 58, pages 1081-1102.

Dexter, R.J. \& Mahmoud H.N. 2004. Predicting Stable Fatigue Crack Propagation in Stiffened Panels, Ship structure Committee SSC-435, Washington.

Dexter, R.J., Mahmoud H.N. \& Pilarski, P. 2005. Propagation of Long Cracks in Stiffened Box-sections under Bending and Stiffened Single Panels under Axial Tension, International Journal of Steel Structures, Volume 5, No. 3, pages 181-188.

Glinka, G. \& Shen, G. 1991. Universal Features of Weight Functions for Cracks in Mode I, Engineering Fracture Mechanics, Volume 10, No. 6, pages 1135-1146.

IACS Common Structural Rules for Double Hull Tankers, Appendix C (consolidated version July 2010); 2012. Available from: http://www.iacs.org.uk/publications.

Koiter, W.T. 1965. Note on the Stress Intensity Factor for Sheet Strips with Cracks under Tensile Loads, Report No 314, University of Technology, Laboratory of Engineering Mechanics, Delft, Netherlands.

Kwon, S., Vassalos, D. \& Mermiris, G. 2010. Adopting a Risk-based Design Methodology for Flooding Survivability and Structural Integrity in Collision/Grounding Accidents., Proceedings of the 11th International Ship Stability Workshop, Wageningen.

Marchal, N., Boutillier, V. \& Serror, M. 2009. Simulation of Behavior of Fatigue Cracks: a Complete Industrial Process on a Typical Connection in a FPSO (Side shell longitudinal stiffener), Bulletin Technique, Bureau Veritas, France.

Serror, M. \& Marchal, N. 2009 Simulation of Behavior of Fatigue Cracks: a Complete Industrial Process on a Typical Connection in a FPSO (Longitudinal stiffener bracket on transverse web), Proceedings of the ASME 200928 th International Conference on Ocean, Offshore and Artic Engineering. Honolulu, Hawaii.

Underwood, J.M., Sobey, A.J., Blake, I.R.J. \& Ajit Shenoi, R. 2012. Ultimate collapse strength assessment of damaged steel-plated structures, Engineering Structures, Volume 38, pages 1-10. 